\title{
High-Load Titanium Drilling Using an Accurate Robotic Machining System
}

\author{
Robert Brownbill $^{1(\otimes)}(\mathbb{D})$, Philip Silk ${ }^{2}$ D, Peter Whiteside $^{1}\left(\mathbb{D}\right.$, Windo Hutabarat $^{3}$ (D), \\ and Harry Burroughes ${ }^{2}$ (iD \\ ${ }^{1}$ Electroimpact UK Ltd., Manor Lane, Hawarden, Deeside CH5 3ST, UK \\ \{robertb, peterw\} @electroimpact.com \\ 2 The Advanced Manufacturing Research Centre - The AMRC, University of Sheffield, \\ Factory 2050, Sheffield S9 1ZA, UK \\ \{p.silk, h. burroughes\} @amrc.co.uk \\ 3 Department of Automatic Control and System Engineering, University of Sheffield, \\ Portobello Street, Sheffield S1 3JD, UK \\ w.hutabaratesheffield.ac.uk
}

\begin{abstract}
Robotic drilling systems have been used in the manufacture of large aerospace components for a number of years. Systems have been developed by several systems integrators in order to accurately drill materials from CFRP to Titanium. These systems, however, have been unable to achieve large diameter holes in Titanium due to reduced structural stiffness and end effector capabilities. Typically, large holes are either drilled using large cartesian CNC-controlled machines or drilled using automated drilling units (ADU). However, there is a pull from aerospace OEMS to move away from large monolithic machines, in favour of flexible robotic system. Flexible robotic systems provide a number of benefits for large structure assembly. The following report primarily outlines drilling trials conducted on the Accurate Robotic Machining System, during which holes from $25 \mathrm{~mm}$ to $32 \mathrm{~mm}$ ID were drilled in titanium implementing an empirical test schedule. Additionally, a discussion on the benefits of drilling large diameter holes using flexible robotic platforms.
\end{abstract}

Keywords: Robotic drilling · Titanium drilling $\cdot$ Robotic drilling systems · Large hole titanium drilling · Heavy Duty Robot $\cdot$ Accurate robotics

\section{Introduction}

Robotic drilling systems have been used in the manufacture of aerospace components for a number of years. There have been systems developed to accurately drill materials such as Aluminium, CFRP and Titanium.

To the best of the authors' knowledge, the largest hole drilled by a robotic drilling system in titanium was $1.0^{\prime \prime}(25.4 \mathrm{~mm})$ in diameter, although this was also carried out under research conditions. Aerospace production holes drilled through Aluminium with robotic drilling systems currently don't typically exceed $5 / 8^{\text {th }}$ ( $(15.88 \mathrm{~mm})$. 
To achieve the hole quality demanded by the aerospace industry, it has been typical that all 'large diameter' holes are either drilled using CNC-controlled machines, expensive bespoke Cartesian drilling machines or drilled manually using automated drilling units (ADUs).

There are huge benefits to using a robotic drilling system over a typical machining centre. The current industry move to 'flexible' and 'elastic' automation cells means that re-tasking or repurposing the automation is essential to ensuring the overall equipment effectiveness (OEE) is maximized through the simple change of the end-effector. This, in most cases, can easily be an automated process requiring no human interaction.

Robotic systems require much simpler civil engineering works and have a much smaller 'footprint' for the same working volume than that of a traditional gantry machine. These benefits coupled with much shorter lead time and reduced overall cost provide a strong business case for investment into this technology.

These systems, however, have been unable to achieve large diameter holes due to the inherent reduced structural stiffness of the robot arm coupled with the limited robot payload. This has meant that end-effector capabilities are typically not up to the same standard as Cartesian CNC Milling Machines.

Large diameter drilling using robots is also challenging due to the positional accuracy of 'off-the-shelf' robot arm being in excess of $\pm 0.5 \mathrm{~mm}$. One way to resolve these problems is by improvements to the feedback system, such as the Electroimpact Accurate package. This Accurate package enable the system to achieve a positional accuracy of $\pm 0.25 \mathrm{~mm}$ or better across the robot's working volume. The increase in accuracy also does not require external metrology equipment feedback. Additionally, this also ensure the robotic system remains accurate for multiple processes, without the requirement for localised datum feature relocation or re-teaching of positions.

Further complications are seen during drilling, whereby the thrust force causes the robot arm to flex, especially when the loads are close to the limits of the robot system. The robot's rigidity is not enough to prevent 'skidding' of the tool tip across the workpiece, which subsequently leads to damage, poor quality parts and hole being drilled far outside of positional tolerances.

Skidding can also be prevalent of the robot nosepiece during clamp up process. As the load is increased through the robot arm - a typical system with no secondary feedback are unable to compensate for the arm flex resulting in the nose-piece skidding on the structure causing damage and requiring expensive rework. The additional scales and enhanced kinematic model guarantee the "anti-skid" technology.

The pressure foot allows the system to remain clamped to the component throughout the drilling cycle and even when full retract pecks are used, there is no danger of movement of the system.

Titanium is used in aircraft structures for high-strength applications in high-stress areas such as around the Engine Pylons and Landing Gear attachment points. Fastener sizes of up to $1.25^{\prime \prime}(31.75 \mathrm{~mm})$ are commonly seen in long-haul aircraft wing structures in these areas. During the manufacture of these wing structures there is the requirement to drill a large number of different holes in multiple stack assemblies. Stack assemblies can consist of multiple layers or 'stacks' of different materials including CFRP, Aluminium 
and Titanium. This assembly process ensures the drilled holes are concentric on final assembly of the components (Fig. 1).

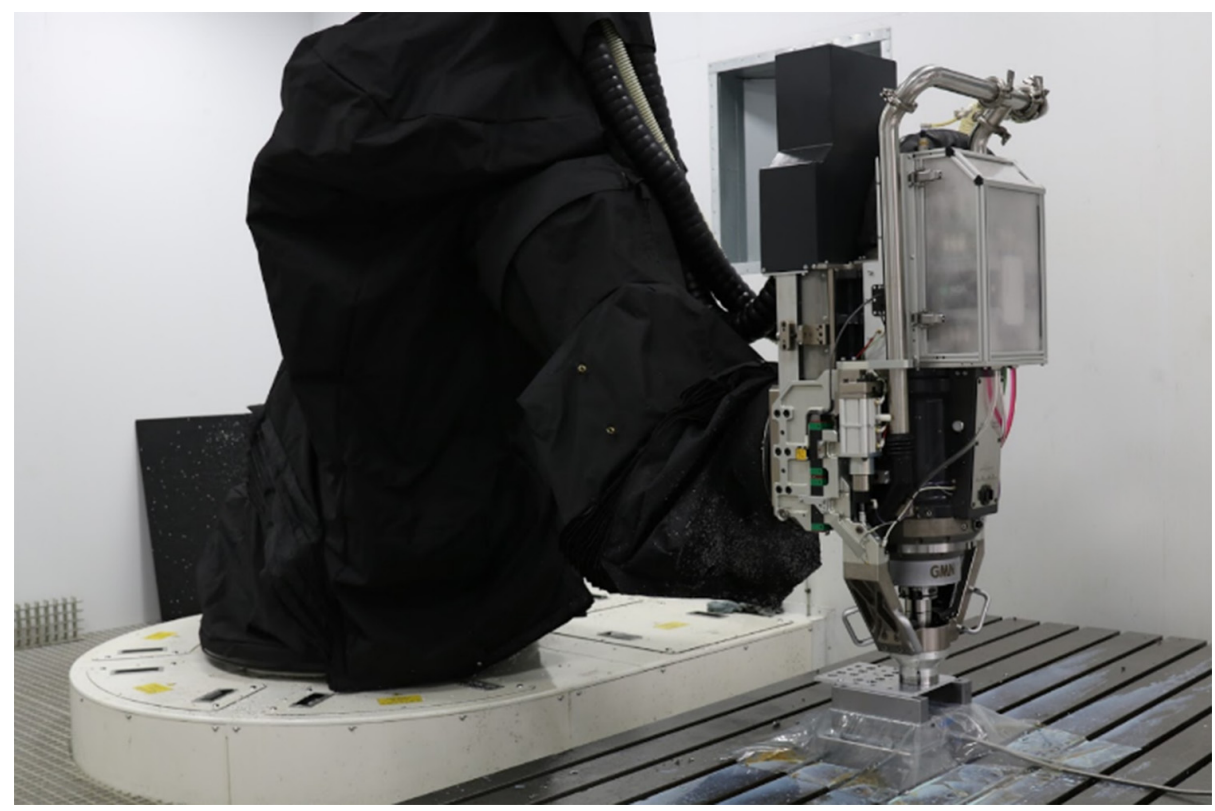

Fig. 1. AMRC accurate robotic machining system

The following report outlines drilling trials conducted using empirical methods on the Accurate Robotic Machining System, during which holes from $25 \mathrm{~mm}$ to $32 \mathrm{~mm}$ diameter were drilled in titanium.

\section{Related Works}

As the size of the assemblies grows, the cost of a traditional gantry-style machine becomes less economical compared to that of a robotic system with the same capabilities [1]. Industry demand for the drilling of large-scale aerospace components, which contain titanium, has increased. Robotic drilling of titanium using serial robots has been attempted but high compliance is a major challenge [2]. Ways to improve the accuracy of robots using auxiliary devices such as external feedback sensors have been proposed [3], but the use of such robots to drill high-load large-diameter holes is not found in literature.

No such works have been found exploring the use of articulated robots for large hole drilling, without the use of external thrust-reducing mechanisms (such as vibration assistance). No internal research was carried out within Electroimpact as there was, until now, no direct request from a potential Customer for that requirement. This paper takes that Customer request and directly addresses their requirements, filling in the knowledge gap of all parties involves into the large-hole pure drilling capability of the robotic system. 


\section{Accurate Robot Architecture}

With the addition of secondary feedback, high-order kinematic model, and a fully integrated conventional CNC control, robotic technology can now compete on a performance level with customized high precision motion platforms. As a result, the articulated arm can be applied to a much broader range of assembly applications that were once limited to custom machines [4].

Although Electroimpact's accurate upgrade is not the only way to increase robot accuracy, it provides multiple advantages over systems such as real-time metrology feedback. As part of the upgrade process from 'off-the-shelf' robot to Accurate Robot, Electroimpact fits high accuracy external scales to every axis of the robot structure (Fig. 3). The Accurate Robot system is controlled via industry standard Siemens 840Dsl $\mathrm{CNC}$ which handles all controls requirements and offers a familiar interface to programmers and operators. Drawing from common axis configuration in machine tool design, the robot arm is integrated by Electroimpact with patented secondary position sensors at the output of each axis.

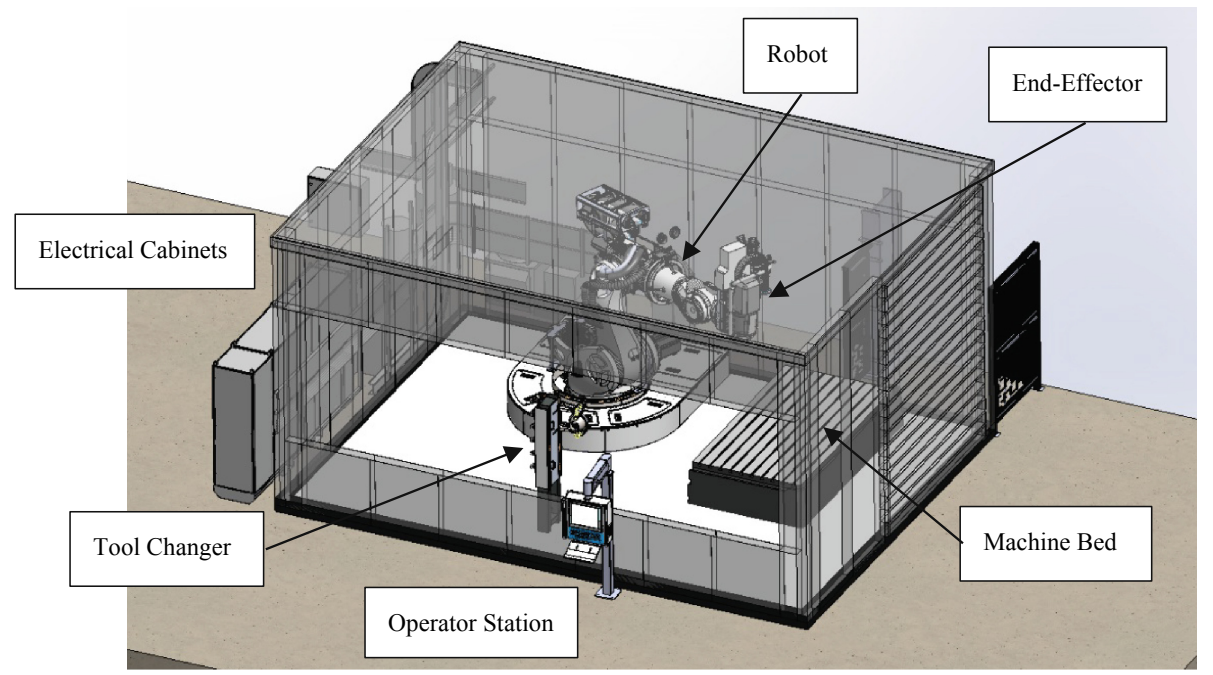

Fig. 2. AMRC factory 2050, ARMS cell layout

The upgrade significantly improves the system enabling calibrated to accuracies to be below $\pm 0.25 \mathrm{~mm}$ over a large global volume. This patented solution has broadened the range of applications for unguided industrial robots in the aerospace industry to include high-precision single and dual-sided drilling and fastening, accurate material removal (trimming, milling), and accurate robotic fibre placement [5] (Fig. 2).

The system implemented at the AMRC utilizes a KUKA Titan as the base architecture before the electronics were upgraded with the Accurate Package.

The end-effector in this application is a fixed spindle with small quill axis $(\sim 60 \mathrm{~mm})$ allowing for milling and drilling operations. 
Although globally, Electroimpact will guarantee accuracies of $\pm 0.25 \mathrm{~mm}$ over the robot's working envelope, the compensation routine was optimized for use in the machining volume over the fixed bed, this has seen the accuracy (for this application) improve to around $\pm 0.17 \mathrm{~mm}$.

ARMS is unique as it is currently, the only one to utilize the Accurate Robot architecture. The robot used has a large working volume and medium load $(750 \mathrm{~kg})$. Other models are available, with shorter reach, increasing the maximum payload but reducing the working volume. Below, details are provided on the modifications carried out to create ARMS.

\subsection{Kinematic Model}

The Electroimpact kinematic model utilizes the secondary feedback of external encoders as well as the robot-specific data to greatly improve accuracy. The kinematic model is able to compensate the robots commanded position for the full working envelope and full working payload of the robot, ensuring that the actual position of the tool-point is always accurate even during high load process.

The patented kinematic model uses the dual feedback of the motor encoder positions and high accuracy secondary scales to adjust the robots commanded position and compensate for structural flex and deflections in the system due to loading.

The compensation routine carried out during installation, is also taken into account in the kinematic model to ensure that the robot is not only highly repeatable but, more importantly, highly accurate within the global reference system.

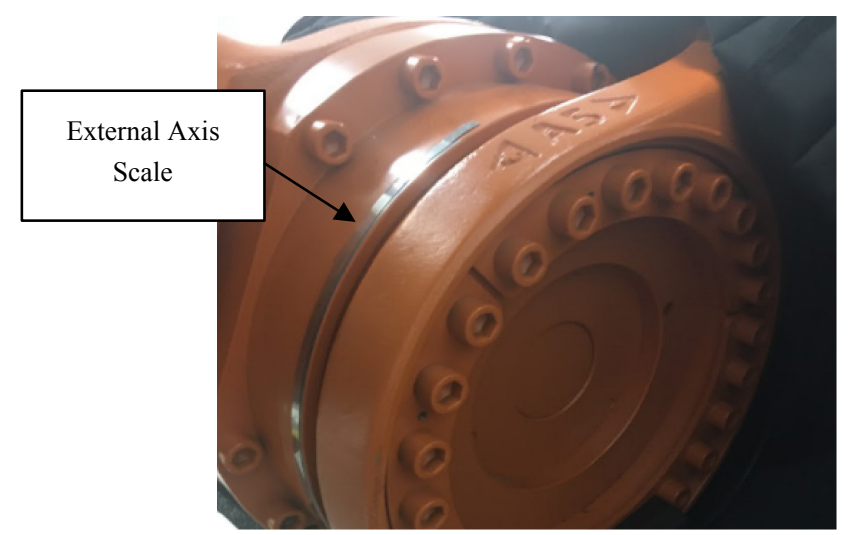

Fig. 3. External axis scale (secondary feedback) showing the encoder strip to gain an accurate position of each joint.

The enhanced kinematic model takes into account the moments of inertia, gravity loads of the arm sections, payload droop and applied (clamp) force deflections to update the Siemens built-in robot kernel for motion. This enables accuracy across the entire working volume. If required, the 'working volume' can be reduced and more data taken over a smaller volume to optimise and improve the accuracy of the system to suit a 
specific application. For ARMS, the accuracy package was optimised over the machine bed working volume.

The deflection model, measured by the scales, allows for real-time compensation from external forces. This uses the control system to effectively remove the backlash effects and gives an (almost) infinitely stiff axis. This will also mean that accuracy and repeatability is greatly improves through an omni-directional routine, rather than approaching a datum point from the same approach direction. Figure 4 shows a real-time snapshot of the commanded versus actual/measured position for the robot system.

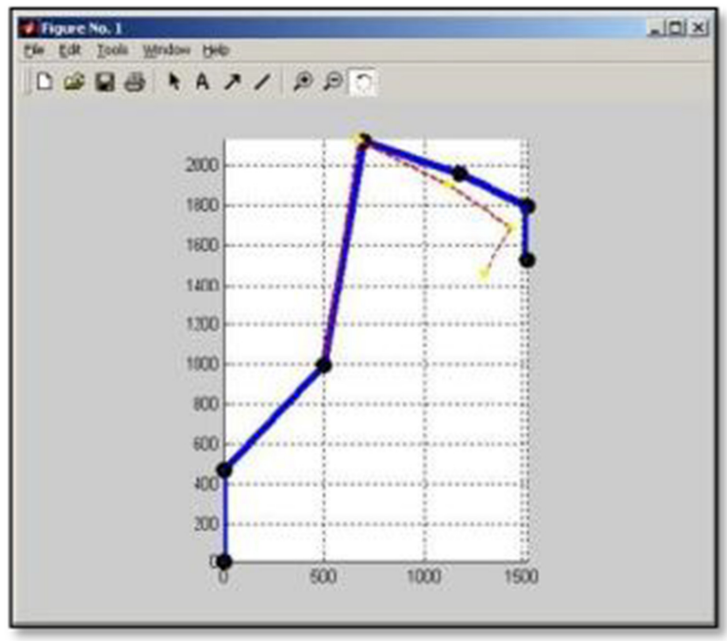

Fig. 4. Kinematic model deflections

\subsection{Spindle}

The spindle chosen by the AMRC was GMN HCS 280 - 18000/60. This was the largest spindle available at the time from GMN allowing for the widest range of capabilities from this system. For a Research Technology Organization (RTO), testing on this system will allow research partners and companies to monitor different parameters (such as spindle torque and thrust load) and enable them to size their own systems appropriately.

\subsection{Pressure Foot}

A removable pressure foot was included in the system to allow for advanced drilling operations. Although the accuracy and effective rigidity of the robot is greatly increased due to the accurate package, it is not sufficient to cope with drilling/peck loading. Therefore, a pressure foot is used to apply pre-load to the workpiece. This preload should be greater than the peak drilling thrust load to prevent movement of the end-effector mid-cycle. Load cells within the pressure foot allow the robot to clamp to a user-defined preload. 
Drill thrust load is also monitored and should the thrust load exceed a certain percentage of the preload, the $\mathrm{CNC}$ will display an alarm - stopping the cycle and preventing damage to the workpiece.

Although active normality correction is common on specific drilling system, there is no such feedback on ARMS. There is only passive normality correction of the pressure foot by means of a swivel nose piece of "cup and cone" type design.

A proximity switch ensures that the controller is aware of the fitment of the pressure foot to prevent collisions and damage to the system.

\subsection{Additional Sensors/Data Sources/Systems}

The system uses additional sensors to ensure better part quality. There are load cells that allow the controller to measure the pressure foot applied force (clamp force). This force can be adjusted as per the user requirements.

There is a laser distance measurement that measures the distance travelled by the pressure foot and controller uses this data to adjust point the drilling tool centre point for the top of stock measurement.

Built into the spindle is a high-accuracy displacement sensor. This is vital and required for the best part quality. As the spindle heats up, the thermal expansion of the components results in a $\mathrm{Z}$ shift that must be accounted for in the controller.

There are also thru-bit and flood coolant system on the end-effector. These were required for the titanium drilling processes. These provide a superior finish on advanced material compared to MQL. Although, the system may be adapted through minor design alterations to accept an MQL unit. The coolant may be disabled, and compressed air diverted to enable an air-blast cooling. The flexibility to choose the coolant technique allows the AMRC and ARMS to tailor their research to suit each clients desired application.

\subsection{Programmable Drilling Parameters}

Up to 16 separate parameters can be adjusted during a drilling procedure in order to optimise the process. The parameters fall under one of the following categories.

- Feeds and speeds: RPM, mm/rev, $\mathrm{mm} / \mathrm{min}$

- Peck cycle: distance and number of pecks

- Allowable spindle thrust force: $\mathrm{max} / \mathrm{min}$

- Auxiliary services: Coolant, air blast

- Pressure foot load

\section{Industrial Applications}

As previously mentioned, robotic drilling solutions have been used in the aerospace industry for a number of years. The robotic system designed and integrated into aerospace production facilities up to date have only had the capability to drill hole which do not 
exceed $5 / 8^{\text {th }}(15.88 \mathrm{~mm})$ in diameter. However, across aerospace facilities there are requirements to drill large holes.

Electroimpact have previously commissioned systems such as Gear Rib Automated Wing Drilling Equipment (GRAWDE), which have been designed to drill holes up to 1.25 inch $(31.75 \mathrm{~mm})$ diameter in the A380 gear rib. This system however, is a Cartesian machine and as such as limited flexibilities and a fix role. A similar Cartesian system produced by Electroimpact, the Horizontal Automated Wing Drilling Equipment (HAWDE), allows for the drilling of wing assemblies with working envelopes of $42 \mathrm{~m} \times$ $8 \mathrm{~m} \times 2 \mathrm{~m}$ [6]. The system is able to accurately position a drilling tool with 5 degrees of freedom. These cartesian systems highlight the need for large volume drilling systems capable of drilling larger holes. Other such cartesian systems have been utilized on other wing-box assembly lines for the latest generation aircraft programs from multiple global manufacturers.

Although, both systems (GRAWDE, HAWDE) are able to meet the drilling requirements of Airbus, they have been specifically designed for their roles. As such the reutilisation of such machinery is difficult and now that the production of the A380 has stop the machinery is likely to be decommissioned, with no further use. Robotic systems are able to provide an alternative to this issue, as reutilisation a capable with the change/ redesign of an end effector.

Developmental robotic systems such as ARMS at the AMRC demonstrate how such robotic architecture can achieve comparable large-hole drilling capability to the more expensive and constrained cartesian-machine counterparts.

\section{Experimental Methods}

ARMS was used to assess the feasibility of using an Electroimpact Accurate Robot to drill aerospace production holes in high-load areas of the aircraft structure. Although metric drills were used, sizes were chosen to best match the equivalent imperial sizes that would be typical of a production system.

Coupons of $45 \mathrm{~mm}$ thick 6Al4V Titanium were prepared to fit in a machinist vice. The test schedule was created to evaluate the system's capabilities with increasing hole diameter through the following sizes:
a) $27.5 \mathrm{~mm}$ One Shot
b) $28.5 \mathrm{~mm}$ Ream (from $27.5 \mathrm{~mm}$ Downsize Hole)
c) $29.5 \mathrm{~mm}$ One Shot
d) $32.0 \mathrm{~mm}$ One Shot

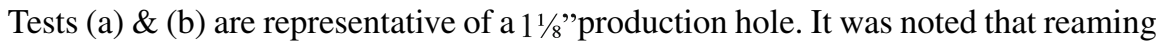
thrust loads and drill torques were much less than the one-shot pre-ream drill cycle. Therefore, tests (c) \& (d) were not reamed. Capability to drill $32.0 \mathrm{~mm}$ one-shot hole is representative of a $1 \frac{1 / 4^{\prime \prime}}{\text { production hole. }}$

Although in most industrial applications a solid carbide twist drill would be used to drill these holes, for the purposes of these drill trials, a Guehring RT800 3D tipped drill was used as a cost-effective, representative example. The benefit of being able to quickly 
change the RT800WP (carbide, FireEX coated) drill tip meant the tool didn't need to be reset in the holder and length re-measured for each size change - drastically speeding up the testing process. The use of this tipped drill will provide a good baseline for feasibility studies for industrial applications, typically, tipped drills require more torque and more thrust loading than their solid carbide counterparts.

Furthers trials, around 12 months later, were carried out using a $27.54 \mathrm{~mm}$ diameter solid carbide twist drill, provided by an Industrial partner to prove process capability. It was observed that the drill thrust loads were approximately 10-20\% lower using the solid carbide twist drill over the tipped cutter.

Successful cycles were run with spindle speed ranges of 100-250 RPM and feed rates of $0.09-0.17 \mathrm{~mm} / \mathrm{rev}$. Clamp load was close to the robot's maximum. A peck-drilling cycle was used with flood and thru-bit coolant active.

A 3-axis Dynamometer was used to measure drilling forces and monitor the process for excessive vibrations. The vice was mounted to the dynamometer interface plate and forces in three dimensions measured.

Hole quality inspected using a CMM at Electroimpact's facility along with a surface roughness indicator.

As ARMS doesn't have local normality correction (as a production drilling robot would have) and the titanium coupons were not perfectly flat, perpendicularity to the top surface of the coupon was not as important as the hole-vector parallelism between all holes on the coupon. This was measured to assess if the drill had a tendency to 'wander' away from the thrust axis.

The edge of the coupon was water cut from a larger titanium plate. With no datum feature, hole position was measured relative to the first hole given the programmed hole spacing.

\section{Results and Discussion}

The Accurate Robotic Machining System was capable of drilling all the holes as detailed in the testing schedule to a very high standard, suitable to meet a typical aerospace manufacturers machine specification (Fig. 5).

The tipped drill has ' $h 7$ ' tolerance and was used as the reaming tool. Although not indicative of an industrial process, all drilling parameters were observed to be well within the capabilities of the system. It was, however, observed that carrying out a 'rapid retract' once the reaming cycle was completed left a spiral score mark within the hole - this could be solved by feeding the tipped drill back out of the hole or using a specific machine reamer.

Testing was repeated during a second session using a tipped machine reamer at the $28.5 \mathrm{~mm}$ diameter (reaming from $27.5 \mathrm{~mm}$ downsize diameter) along with drilling using a full-length solid carbide production cutter. Limited cycles were run using these cutters to reduce unnecessary wear. Eight holes were drilled $27.5 \mathrm{~mm}$ one shot, three were reamed thru and one was partially reamed (to measure concentricity - the systems ability to return to the same hole). The scoring that can be seen in hole \#1 (top right) was due to a rapid retract command as part of the ream cycle - this was then changed to a feed retract on the subsequent two and a half holes and excellent surface finish results were seen (Fig. 6). 


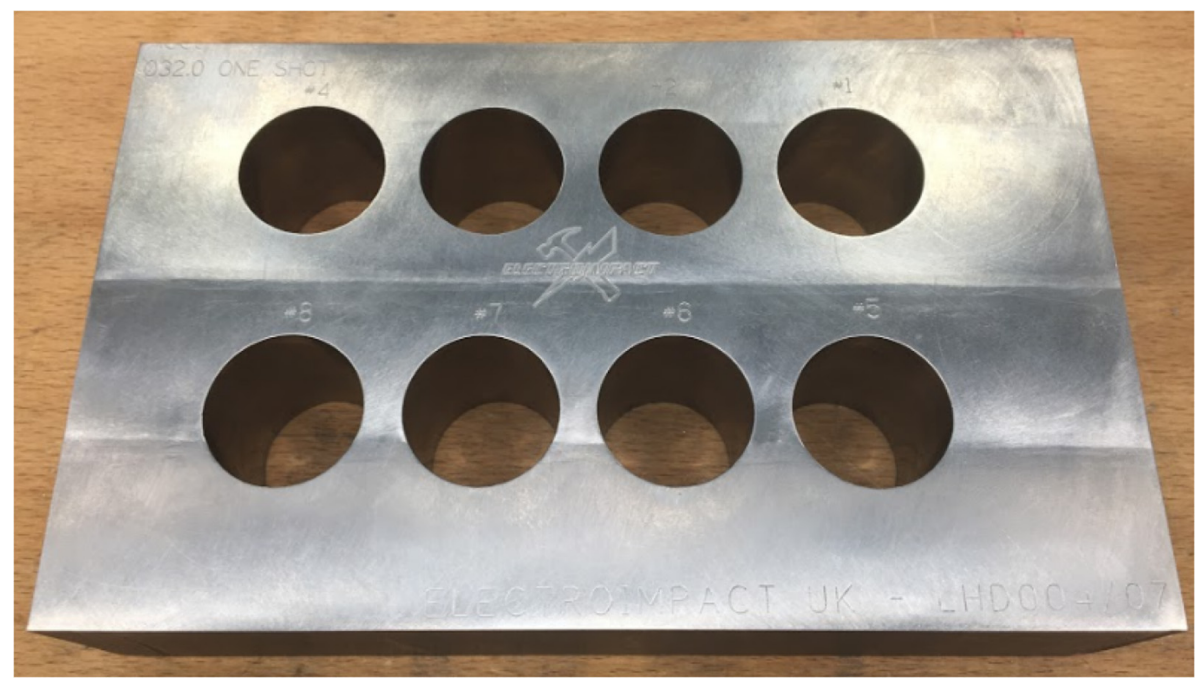

Fig. 5. $32.0 \mathrm{~mm}$ test coupon a (guehring tipped drill)

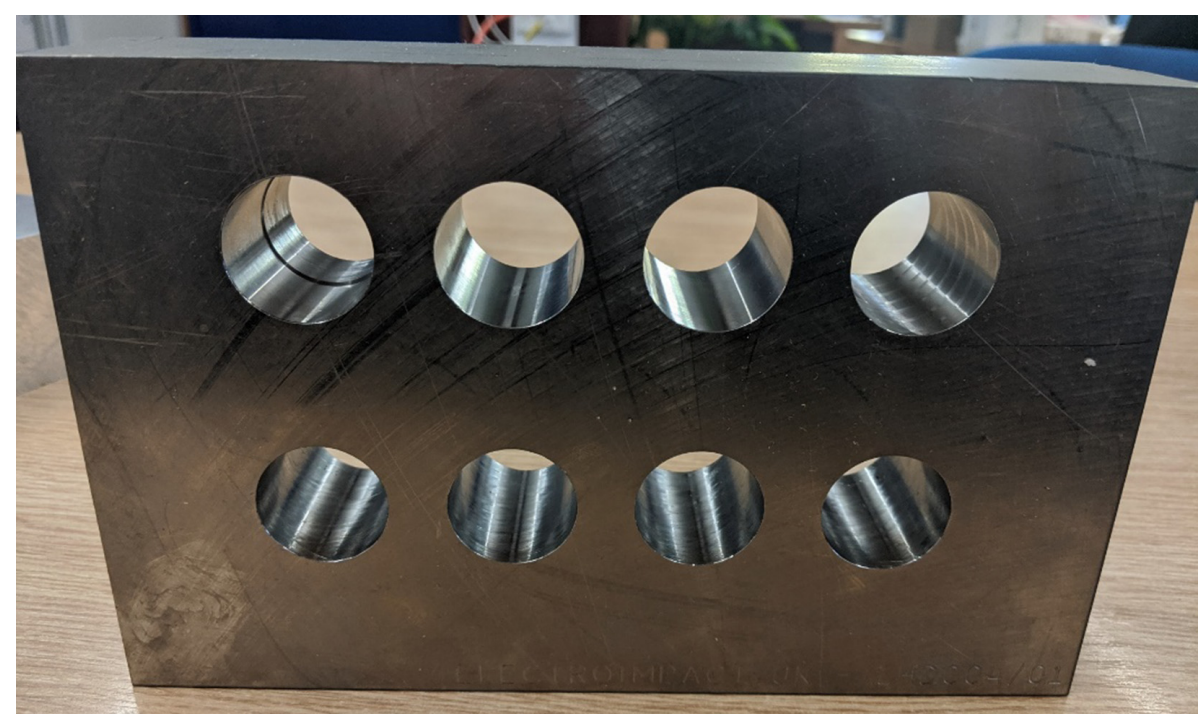

Fig. 6. Production cutter coupon drill and ream holes (holes 1,2,3: One-shot drill $27.5 \mathrm{~mm} \&$ Ream 28.5 mm, hole 4: Partial ream, holes 5,6,7,8: One-shot drill $27.5 \mathrm{~mm}$ )

\subsection{Dynamometer Results}

Good, consistent results were seen during the drilling process with no unwanted vibration observed (Fig. 7). 


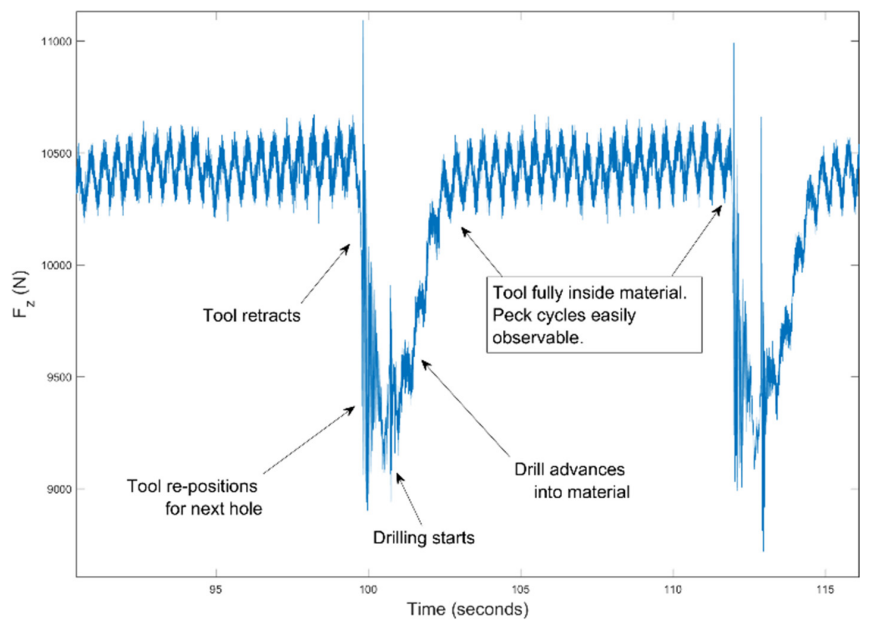

Fig. 7. Dynamometer force results

\subsection{Hole Quality}

Results were collated from 48 holes (in six coupons) as detailed in the test schedule. The above table of results is the range observed across all coupons and would meet a typical Aerospace Industry specification for holes of this diameter (Table 1).

Table 1. Collated results of the trial using tipped cutters.

\begin{tabular}{l|l}
\hline Quality metric & Result \\
\hline Hole position & $\pm 0.25 \mathrm{~mm}$ \\
\hline Diameter (drill) & $+0 /+46 \mu \mathrm{m}$ \\
\hline Diameter (ream) & $-23 /-18 \mu \mathrm{m}^{*}$ \\
\hline Hole cylindricity & $47 \mu \mathrm{m}$ \\
\hline Hole vector parallelism (to Hole 1$)$ & $0.15 \mathrm{~mm}$ \\
\hline Hole surface finish quality (drill) & $<1.4 \mu \mathrm{m} \mathrm{Ra}$ \\
\hline Hole surface finish quality (ream) & $<0.8 \mu \mathrm{m} \mathrm{Ra}$ \\
\hline
\end{tabular}

*Measurements fall within the tolerance band of the 'h7' cutter.

In all cases, ARMS was able to achieve the $32.0 \mathrm{~mm}\left(1.260^{\prime \prime}\right)$ diameter holes, drilled one-shot through Titanium. Spindle torque loading was not observed to exceed $50 \%$ throughout drilling cycles, with no greater than $25 \%$ torque observed for the reaming cycles (Table 2).

The results from the limited trial of the production cutters and reamers show the improvements in cylindricity, surface finish quality and vector parallelism as expected 
Table 2. Collated results of the trial using production cutters $(27.5 \mathrm{~mm} / 28.5 \mathrm{~mm}$ diameters only).

\begin{tabular}{l|l}
\hline Quality metric & Result \\
\hline Hole position & $\pm 0.25 \mathrm{~mm}$ \\
\hline Diameter (drill) & $-3 /+10 \mu \mathrm{m}$ \\
\hline Diameter (Ream) & $0 /-+3 \mu \mathrm{m}$ \\
\hline Hole cylindricity (Ream) & $16 \mu \mathrm{m}$ \\
\hline Hole vector parallelism (to Hole 1) & $0.019 \mathrm{~mm}$ \\
\hline Hole\#4 Concentricity (Drill/ream) & $0.026 \mathrm{~mm}$ \\
Hole surface finish quality (drill) & $<1.3 \mu \mathrm{m} \mathrm{Ra}$ \\
\hline Hole surface finish quality (ream) & $<0.45 \mu \mathrm{m} \mathrm{Ra}$ \\
\hline
\end{tabular}

from the use of a solid carbide cutter. The increased stiffness of the tools and use of a reaming cutter far exceeded the production requirements that were being assessed.

Spindle torque values were observed to be comparable to that of the tipped cutters with a minor increase for the first drilled holes, as this was a brand-new cutter. Torque values were observed to return to those observed previously after the second hole.

The limiting factor throughout these trials was the pre-load clamp force available to counter the drill thrust loading. This was close to the Robot's maximum for the largest holes drilled.

\section{Conclusions}

Confidence has been proven that the Electroimpact Accurate Robot architecture is capable of meeting aerospace production requirements for high-load fastener holes of up to $1 \frac{1}{4}$ " diameter. However, this has been conducted, both in a favourable Robot pose and under research condition.

ARMS is reaching the limits of its advanced material drilling capability due to the limits of the clamping pre-load available. Should the drilling of larger holes be required, additional measures to reduce drill thrust load would be necessary.

The Electroimpact Accurate Robot package providing the controls-based stiffness has enabled this system to do something previously thought not possible.

The focus of future work will be to reduce the clamp up and thrust force required to produce a large diameter hole in Titanium, primarily through process optimisation. This will allow large diameter holes to be drilled where fixturing, orientation and access are not optimum or vary in the case of production setups.

Acknowledgements. We gratefully acknowledge the support of Royal Academy of Engineering, Advanced Manufacturing Research Centre, Electroimpact UK Ltd, The University of Sheffield, HVM Catapult and Aerospace Technology Institute. 


\section{References}

1. Verl, A., Valente, A., Melkote, S., Brecher, C., Ozturk, E., Tunc, L.T.: Robots in machining. CIRP Ann. 68(2), 799-822 (2019)

2. Kim, S.H., et al.: Robotic machining: a review of recent progress. Int. J. Precis. Eng. Manuf. 20(9), 1629-1642 (2019)

3. Bi, S., Liang, J.: Robotic drilling system for titanium structures. Int. J. Adv. Manuf. Technol. 54(5), 767-774 (2011)

4. Saund, B., DeVlieg, R.: High accuracy articulated robots with CNC control systems. SAE Int. J. Aerosp. 6(2), 780-784 (2013)

5. DeVlieg, R.: Accurate robot technology (2019). https://electroimpact.com/Products/Robots/ Overview.aspx. Accessed on 19 Dec 2019

6. Calawa, R., et al.: HAWDE Five Axis Wing Surface Drilling Machine (2004). https://www.ele ctroimpact.com/WhitePapers/2004-01-2806.pdf. Accessed on 29 July 2020

Open Access This chapter is licensed under the terms of the Creative Commons Attribution 4.0 International License (http://creativecommons.org/licenses/by/4.0/), which permits use, sharing, adaptation, distribution and reproduction in any medium or format, as long as you give appropriate credit to the original author(s) and the source, provide a link to the Creative Commons license and indicate if changes were made.

The images or other third party material in this chapter are included in the chapter's Creative Commons license, unless indicated otherwise in a credit line to the material. If material is not included in the chapter's Creative Commons license and your intended use is not permitted by statutory regulation or exceeds the permitted use, you will need to obtain permission directly from the copyright holder.

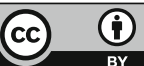

